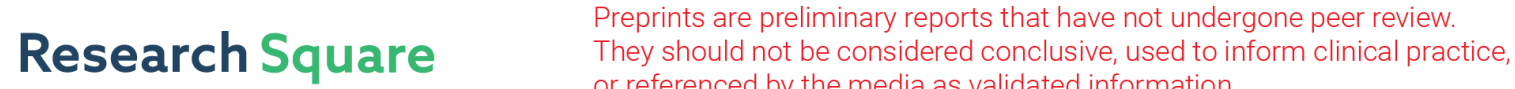 or referenced by the media as validated information. \\ Inhaled Nitric Oxide for postexposure chemoprophylaxis of COVID-19
}

Antoine AbdelMassih ( $\square$ antoine.abdelmassih@kasralainy.edu.eg )

Cairo university

\section{Rafeef Hozaien}

Meryam El Shershaby

Aya Kamel

Habiba-Allah Ismail

Mariem Arsanyous

Nadine El-Husseiny

Noha Khalil

Youstina Naeem

Raghda Fouda

\section{Systematic Review}

Keywords: Inhaled Nitric Oxide, COVID-19, Furin Inhibition, Postexposure prophylaxis

Posted Date: June 14th, 2021

DOI: https://doi.org/10.21203/rs.3.rs-612293/v1

License: (c) (i) This work is licensed under a Creative Commons Attribution 4.0 International License. Read Full License 


\section{Abstract}

\section{Background:}

Postexposure prophylaxis has been an overlooked strategy in the context of COVID-19. Inhaled Nitric Oxide offers itself as a potential tool in this context. The aim of this systematic review was to depict previous in vivo and in vitro studies demonstrating an antiviral role for NO

\section{Methodology:}

Embase, Medline and the Cochrane Central Register were used to search for specific keywords such as "Nitric oxide" AND "Antiviral activity" for relevant publications up to $1^{\text {st }}$ of June 2021. The systematic review was performed using PRISMA protocol

\section{Results:}

Twenty-one studies were identified depicting an antiviral role for Nitric Oxide. Those studies involved sixteen viruses. Only four of the depicted studies were clinical trials, while three were performed on a murine model. The remainder of the studies involved in vitro experimentation of the role of $\mathrm{NO}$ in halting viral replication of several viruses including SARS-CoV-2

\section{Conclusion:}

While early reports of NO role in the treatment of COVID-19 suggested its use for the treatment of established ARDS, NO seems to have a much earlier and more efficient prophylactic role. It inhibits a protease needed for canonical viral replication of SARS-CoV-2, namely Furin, by decreasing calcium's cytosolic levels. This might add a significant tool for postexposure chemoprophylaxis in the at-risk group, especially medical personnel.

\section{Introduction}

SaNOtize (Canada (Vancouver-based/ NCT04443868 biotech firm) recently created a self-administered nitric oxide nasal spray (NONS) that is said to potentially reduce Coronavirus Disease 2019 (COVID-19) viral load in recently infected patients. After completing early-stage clinical trials in Canada and the United Kingdome (UK), SaNOtize, Ashford and St. Peter's Hospitals, the National Hospital System (NHS) foundation, and a few pathology services in the UK announced the results of Phase II trials. The results indicate that NONS can be a powerful and safe antiviral treatment. It could prevent COVID-19 transmission, shorten its duration and reduce the severity of its symptoms.

Several reports have discussed Nitric oxide use in the context of COVID-19. Lotz et al., for example, highlighted its potential to improve acute respiratory distress syndrome in COVID-19. However, the aforementioned clinical trial results suggest that it has a much earlier antiviral role in COVID-19. The exact mechanism of how it executes this role will be discussed in this report.(1) 


\section{Methodology}

Embase, Medline and the Cochrane Central Register were used to search for specific keywords such as "Nitric oxide" AND "Antiviral activity" for relevant publications up to $1^{\text {st }}$ of June 2021. The systematic review was performed using PRISMA protocol. Study Selection criteria Population: No specific age group or sex Intervention: Nitric oxide Comparison: No comparison has been a purpose of the study Outcome: Antiviral activity (in vivo or in vitro)

\section{Results}

Twenty-one studies were identified depicting an antiviral role for Nitric Oxide. Those studies involved sixteen viruses. Only four of the depicted studies were clinical trials, while three were performed on a murine model. The remainder of the studies involved in vitro experimentation of the role of NO in halting viral replication of several viruses including SARS-CoV-2.

Table 1 exposes the clinical and laboratory trials which used NO as an antiviral agent)(4-24)

\section{Discussion}

-Protease, a critical role in the determination of viral load of COVID-19

One of the very prominent members of the PCSK (pro-protein convertase subtilizing/Kexin) family is the Furin enzyme. Furin is a type 1 membrane-bound protease utilized by multiple pathogens, including Human Immune Deficiency Virus (HIV), Ebola virus, Marburg virus, Severe Acute Respiratory Syndrome Coronavirus 2 (SARS-CoV-2), and even some bacterial toxins. It is believed that the pathogenicity of several viruses can increase several folds once they react with Furin and other Pro-protein convertases. This mechanism revolves around the activation of latent precursor proteins into their active products following Furin cleavage. Hence, Furin-dependent infections may respond to therapeutics targeting host cell Furin. (2)

As previously mentioned, the spike protein of SARS-CoV-2 is the cleavage site of Furin, which plays an essential role in the host range as well as viral infectivity and pathogenesis. As one of the subtilisin-like proteases, Furin requires a polybasic rather than a monobasic cleavage site; hence, cleavage occurs at the junction of the two polybasic subunits of the spike, S1 and S2. The difference in pathogenicity between highly virulent and less virulent influenza strains sets an excellent example of the relationship between viral pathogenicity and the biochemically different cleavage sites.(3)

\section{-How viral protease activity is inhibited by intracellular cations and how NO increases such cations}

Furin is a cellular enzymatic protein that is expressed from the FURIN gene in humans. It has been stated that Furin shows an intriguing interplay with intracellular ions, especially cations. Potassium ions are the most common intracellular ions in our bodies, and magnesium falls in the second rank, where it reveals 
its direct correlation in activating Furin. Molloy et al. specified that calcium level, where Furin can be classified as a "Calcium-dependent enzyme, noticeably influence the activity of Furin. (25)

Moreover, Yamada and colleagues have added further demonstrations supporting the interrelation between Furin and calcium levels. The latter postulation is proved by the effect of Furin hindrance in restricting the escalation of neuronal damage caused by calcium influx consecutive to hypoxic injury. (26) Hence, the impediment of calcium channels can be a promising approach in tackling the rapid augmentation of Furin-activated organisms. Additionally, Li et al. stated in 2019 that Calcium Channel Blockers (CCB) play a novel role in halting the intensity of fever spikes episodes and the thrombocytopenic syndrome, categorized as manifestations of tick-borne hemorrhagic fever.(27)

Surprisingly, NO has been found to encourage calcium efflux from the cells leading to decreased intracellular calcium levels. This has been extensively studied in the context of the vasodilator action of NO. Van Hove et al. proved that NO stimulates Smooth Muscle Cells (SMCs) to relax directly and/or indirectly by removing the elevated calcium. (28)

This might signify that NO can inhibit Furin's action by decreasing cytosolic levels of calcium.

\section{-Postexposure prophylaxis by inhaled NO}

Argyropoulos et al. concluded that diagnostic viral load has no prognostic utility in terms of outcome prediction.(29) There are still conflicting data about this issue; Silva and colleagues in a more recent report, found the saliva viral loads to be significantly higher in patients with chronic respiratory conditions, cardiovascular conditions, kidney disease, and diseases that compromise the immune system. (30) Patients with four or more risk factors had much higher saliva viral loads than patients with fewer risk factors, as did male patients. However, there was no relation between nose and throat viral loads and the risk factors. Saliva viral loads were also higher in patients with worse clinical outcomes. This might signify that early interruption of viral replication in the upper respiratory tract might abort the development of significant symptoms and complications. This rationale might have led to the current inclusion criteria of the ongoing clinical trial for SaNOtize, which involves early administration of the intranasal medication within 48 hours of diagnosis. The administration of SaNOtize might extend later to medical personnel after a documented exposure to an infected case called post-exposure chemoprophylaxis.

\section{Conclusion}

While early reports of NO role in the treatment of COVID-19 suggested its use for the treatment of established ARDS, NO seems to have a much earlier and more efficient prophylactic role. It inhibits a protease needed for canonical viral replication of SARS-CoV-2, namely Furin, by decreasing calcium's cytosolic levels. This action can prevent the exponential increase of viral load in the upper respiratory tract leading to abortion of clinically symptomatic infection and subsequent complication. This might 
add a significant tool for postexposure chemoprophylaxis in the at-risk group, especially medical personnel.

Figure 1 summarizes the antiviral effect of NO and its possible uses in the context of COVID-19

\section{Declarations}

Competing interests: The authors declare no competing interests.

\section{References}

1. Lotz C, Muellenbach RM, Meybohm P, Mutlak H, Lepper PM, Rolfes C, Peivandi A, Stumpner J, Kredel M, Kranke P, Torje I, Reyher C. Effects of inhaled nitric oxide in COVID-19-induced ARDS - Is it worthwhile? Acta Anaesthesiol Scand [Internet]. 2021 May 20;65(5):629-32. Available from: https://onlinelibrary.wiley.com/doi/10.1111/aas.13757

2. AbdelMassih AF, Ye J, Kamel A, Mishriky F, Ismail HA, Ragab HA, El Qadi L, Malak L, Abdu M, ElHusseiny M, Ashraf M, Hafez N, AlShehry N, El-Husseiny N, AbdelRaouf N, Shebl N, Hafez N, Youssef N, Afdal P, Hozaien R, Menshawey R, Saeed R, Fouda R. A multicenter consensus: A role of furin in the endothelial tropism in obese patients with COVID-19 infection. Obesity Medicine. 2020.

3. Hoffmann M, Kleine-Weber H, Schroeder S, Krüger N, Herrler T, Erichsen S, Schiergens TS, Herrler G, Wu N-H, Nitsche A, Müller MA, Drosten C, Pöhlmann S. SARS-CoV-2 Cell Entry Depends on ACE2 and TMPRSS2 and Is Blocked by a Clinically Proven Protease Inhibitor. Cell. 2020;

4. Åkerström S, Mousavi-Jazi M, Klingström J, Leijon M, Lundkvist A, Mirazimi A. Nitric Oxide Inhibits the Replication Cycle of Severe Acute Respiratory Syndrome Coronavirus. J Virol [Internet]. 2005 Feb;79(3):1966-9. Available from: https://journals.asm.org/doi/10.1128/JVI.79.3.1966-1969.2005

5. Keyaerts E, Vijgen L, Chen L, Maes P, Hedenstierna G, Van Ranst M. Inhibition of SARS-coronavirus infection in vitro by S-nitroso-N-acetylpenicillamine, a nitric oxide donor compound. Int J Infect Dis [Internet]. 2004 Jul;8(4):223-6. Available from:

https://linkinghub.elsevier.com/retrieve/pii/S1201971204000529

6. Tang DJ, Xu YH, Dai D, Han YJ, Wang BC, Lang YM, Liang Y, Zeng Y. Clinical analysis of four Chinese hemophiliacs with human immunodeficiency virus infection. Chin Med J (Engl) [Internet]. 1989 Nov;102(11):819-24. Available from: http://www.ncbi.nlm.nih.gov/pubmed/2517721

7. Sanders SP, Siekierski ES, Porter JD, Richards SM, Proud D. Nitric oxide inhibits rhinovirus-induced cytokine production and viral replication in a human respiratory epithelial cell line. J Virol [Internet]. 1998 Feb;72(2):934-42. Available from: http://www.ncbi.nlm.nih.gov/pubmed/9444985 
8. Griffon B, Cillard J, Chevanne M, Morel I, Cillard P, Sergent O. Macrophage-induced inhibition of nitric oxide production in primary rat hepatocyte cultures via prostaglandin E2 release. Hepatology [Internet]. 1998 Nov;28(5):1300-8. Available from: http://doi.wiley.com/10.1002/hep.510280519

9. Takhampunya R, Padmanabhan R, Ubol S. Antiviral action of nitric oxide on dengue virus type 2 replication. J Gen Virol [Internet]. 2006 Oct 1;87(10):3003-11. Available from:

https://www.microbiologyresearch.org/content/journal/jgv/10.1099/vir.0.81880-0

10. Croen KD. Evidence for antiviral effect of nitric oxide. Inhibition of herpes simplex virus type 1 replication. J Clin Invest [Internet]. 1993 Jun;91(6):2446-52. Available from: http://www.ncbi.nlm.nih.gov/pubmed/8390481

11. Simon M, Falk KI, Lundkvist $\AA$, Mirazimi A. Exogenous nitric oxide inhibits Crimean Congo hemorrhagic fever virus. Virus Res [Internet]. 2006 Sep;120(1-2):184-90. Available from: https://linkinghub.elsevier.com/retrieve/pii/S016817020600092X

12. Domachowske JB. Replication of respiratory syncytial virus is inhibited in target cells generating nitric oxide in situ. Front Biosci [Internet]. 2003;8(1):986. Available from:

https://fbscience.com/Landmark/articles/10.2741/986

13. Banerjee NS, Moore DW, Wang H-K, Broker TR, Chow LT. NVN1000, a novel nitric oxide-releasing compound, inhibits HPV-18 virus production by interfering with E6 and E7 oncoprotein functions. Antiviral Res [Internet]. 2019 Oct;170:104559. Available from: http://www.ncbi.nlm.nih.gov/pubmed/104559

14. Bi Z, Reiss CS. Inhibition of vesicular stomatitis virus infection by nitric oxide. J Virol. 1995;69(4):2208-13.

15. Ormerod AD, White MI, Shah SA, Benjamin N. Molluscum contagiosum effectively treated with a topical acidified nitrite, nitric oxide liberating cream. Br J Dermatol [Internet]. 1999 Dec;141(6):1051-3. Available from: http://www.ncbi.nlm.nih.gov/pubmed/10606851

16. Lei C, Su B, Dong H, Fakhr BS, Grassi LG, Di Fenza R, Gianni S, Pinciroli R, Vassena E, Morais CCA, Bellavia A, Spina S, Kacmarek R, Berra L. Protocol for a randomized controlled trial testing inhaled nitric oxide therapy in spontaneously breathing patients with COVID-19. medRxiv Prepr Serv Heal Sci. 2020;

17. Klingström J, Åkerström S, Hardestam J, Stoltz M, Simon M, Falk KI, Mirazimi A, Rottenberg M, Lundkvist $\AA$. Nitric oxide and peroxynitrite have different antiviral effects against hantavirus replication and free mature virions. Eur J Immunol [Internet]. 2006 Oct;36(10):2649-57. Available from: http://doi.wiley.com/10.1002/eji.200535587

18. Lei C, Su B, Dong H, Bellavia A, Di Fenza R, Fakhr BS, Gianni S, Grassi LG, Pinciroli R, Vassena E, Berra L. Protocol of a randomized controlled trial testing inhaled Nitric Oxide in mechanically ventilated 
patients with severe acute respiratory syndrome in COVID-19 (SARS-CoV-2). medRxiv Prepr Serv Heal Sci. 2020;

19. Akaberi D, Krambrich J, Ling J, Luni C, Hedenstierna G, Järhult JD, Lennerstrand J, Lundkvist Å. Mitigation of the replication of SARS-CoV-2 by nitric oxide in vitro. Redox Biol [Internet]. 2020 Oct;37:101734. Available from: https://linkinghub.elsevier.com/retrieve/pii/S2213231720309393

20. Saura M, Zaragoza C, McMillan A, Quick RA, Hohenadl C, Lowenstein JM, Lowenstein CJ. An Antiviral Mechanism of Nitric Oxide. Immunity [Internet]. 1999 Jan;10(1):21-8. Available from: https://linkinghub.elsevier.com/retrieve/pii/S1074761300800035

21. Zell R, Markgraf R, Schmidtke M, Gorlach M, Stelzner A, Henke A, Sigusch HH, Gl ck B. Nitric oxide donors inhibit the coxsackievirus $\mathrm{B} 3$ proteinases $2 \mathrm{~A}$ and $3 \mathrm{C}$ in vitro, virus production in cells, and signs of myocarditis in virus-infected mice. Med Microbiol Immunol [Internet]. 2004 May 1;193(2-3):91-100. Available from: http://link.springer.com/10.1007/s00430-003-0198-6

22. Regev-Shoshani G, Vimalanathan S, McMullin B, Road J, Av-Gay Y, Miller C. Gaseous nitric oxide reduces influenza infectivity in vitro. Nitric oxide Biol Chem [Internet]. 2013 May 31;31:48-53. Available from: http://www.ncbi.nlm.nih.gov/pubmed/23562771

23. Rimmelzwaan GF, Baars MM, de Lijster P, Fouchier RA, Osterhaus AD. Inhibition of influenza virus replication by nitric oxide. J Virol [Internet]. 1999 Oct;73(10):8880-3. Available from: http://www.ncbi.nlm.nih.gov/pubmed/10482647

24. Lin YL, Huang YL, Ma SH, Yeh CT, Chiou SY, Chen LK, Liao CL. Inhibition of Japanese encephalitis virus infection by nitric oxide: antiviral effect of nitric oxide on RNA virus replication. J Virol [Internet]. 1997 Jul;71(7):5227-35. Available from: http://www.ncbi.nlm.nih.gov/pubmed/9188590

25. Molloy SS, Bresnahan PA, Leppla SH, Klimpel KR, Thomas G. Human furin is a calcium-dependent serine endoprotease that recognizes the sequence Arg-X-X-Arg and efficiently cleaves anthrax toxin protective antigen. J Biol Chem. 1992;267(23):16396-402.

26. Yamada M, Hayashi H, Yuuki M, Matsushima N, Yuan B, Takagi N. Furin inhibitor protects against neuronal cell death induced by activated NMDA receptors. Sci Rep. 2018;8(1).

27. Li H, Zhang LK, Li SF, Zhang SF, Wan WW, Zhang YL, Xin QL, Dai K, Hu YY, Wang ZB, Zhu XT, Fang YJ, Cui N, Zhang PH, Yuan C, Lu Q Bin, Bai JY, Deng F, Xiao GF, Liu W, Peng K. Calcium channel blockers reduce severe fever with thrombocytopenia syndrome virus (SFTSV) related fatality. Cell Res [Internet]. 2019;29(9):739-53. Available from: http://dx.doi.org/10.1038/s41422-019-0214-z

28. Van Hove CE, Van der Donckt C, Herman AG, Bult H, Fransen P. Vasodilator efficacy of nitric oxide depends on mechanisms of intracellular calcium mobilization in mouse aortic smooth muscle cells. $\mathrm{Br} \mathrm{J}$ 
Pharmacol [Internet]. 2009 Oct;158(3):920-30. Available from:

http://www.ncbi.nlm.nih.gov/pubmed/19788496

29. Argyropoulos K V., Serrano A, Hu J, Black M, Feng X, Shen G, Call M, Kim MJ, Lytle A, Belovarac B, Vougiouklakis T, Lin LH, Moran U, Heguy A, Troxel A, Snuderl M, Osman I, Cotzia P, Jour G. Association of Initial Viral Load in Severe Acute Respiratory Syndrome Coronavirus 2 (SARS-CoV-2) Patients with Outcome and Symptoms. Am J Pathol [Internet]. 2020 Sep;190(9):1881-7. Available from: https://linkinghub.elsevier.com/retrieve/pii/S000294402030328X

30. Silva J, Lucas C, Sundaram M, Israelow B, Wong P, Klein J, Tokuyama M, Lu P, Venkataraman A, Liu F, Mao T, Oh JE, Park A, Casanovas-Massana A, Vogels CBF, Muenker CM, Zell J, Fournier JB, Campbell M, Chiorazzi M, Ruiz Fuentes E, Petrone M, Kalinich CC, Ott IM, Watkins A, Moore AJ, Nakahata MI, Grubaugh ND, Farhadian S, Dela Cruz C, Ko A, Schulz WL, Ring AM, Ma S, Omer S, Wyllie AL, Iwasaki A. Saliva viral load is a dynamic unifying correlate of COVID-19 severity and mortality. medRxiv Prepr Serv Heal Sci [Internet]. 2021 Jan 10; Available from: http://www.ncbi.nlm.nih.gov/pubmed/33442706

\section{Table}


Table 1: Review of in vivo and in vitro studies of the antiviral effect of Nitric Oxide

\begin{tabular}{|c|c|c|c|c|}
\hline $\begin{array}{l}\text { Reference } \\
\text { number } \\
\text { in text }\end{array}$ & Virus & $\begin{array}{l}\text { Type of } \\
\text { Nitric } \\
\text { oxide } \\
\text { Therapy }\end{array}$ & $\begin{array}{l}\text { Study } \\
\text { Model }\end{array}$ & Main outcome \\
\hline
\end{tabular}

\begin{tabular}{|c|c|c|c|c|}
\hline \multirow[t]{2}{*}{4,5} & \multirow[t]{2}{*}{ SARS-CoV } & $\begin{array}{l}\text { NO donor, } \\
\text { SNAP }\end{array}$ & In vitro & $\begin{array}{l}\text { Inhibited SARS CoV replication cycle } \\
\text { in a concentration-dependent } \\
\text { manner(1) }\end{array}$ \\
\hline & & $\begin{array}{l}\text { NO } \\
\text { donors, } \\
\text { SNAP \& } \\
\text { SNP }\end{array}$ & In vitro & $\begin{array}{l}\text { SNAP \&SNP inhibited the SARS CoV } \\
\text { viral cytopathic effect( } 2 \text { ) }\end{array}$ \\
\hline
\end{tabular}

$17,18,19$

SARS-CoV-2

$\begin{array}{ll}\text { inhaled } & \text { multicenter } \\ \text { NO } & \text { randomized } \\ & \text { controlled } \\ & \text { trial }\end{array}$

Ongoing, antiviral effect of high concentrations of inhaled NO administered during early phases of COVID-19 on spontaneous breathing patients, effect on disease progression.(3)

Ongoing, testing inhaled Nitric Oxide in mechanically ventilated patients with severe acute respiratory syndrome in COVID-19 (SARS-CoV-2). (4)

\begin{tabular}{|c|c|c|c|c|}
\hline & & & $\begin{array}{l}\text { single } \\
\text { center, } \\
\text { randomized } \\
(1: 1) \\
\text { controlled, } \\
\text { parallel-arm } \\
\text { clinical trial }\end{array}$ & $\begin{array}{l}\text { Ongoing, prophylactic therapy to } \\
\text { reduce the instance of COVID-19 } \\
\text { disease in healthcare workers(4) }\end{array}$ \\
\hline & & SNAP & In vitro & $\begin{array}{l}\text { SNAP delayed or completely } \\
\text { prevented the development of viral } \\
\text { cytopathic effect (5) }\end{array}$ \\
\hline \multirow[t]{5}{*}{21} & \multirow[t]{5}{*}{ Coxsackievirus } & $\begin{array}{l}\text { NO donors } \\
\text { SNAP }\end{array}$ & \multirow{5}{*}{$\begin{array}{l}\text { In vitro } \\
\text { Murine } \\
\text { model }\end{array}$} & $\begin{array}{l}\text { NO inhibits CVB3 replication by } \\
\text { inhibiting protease activity and } \\
\text { interrupting the viral life cycle (6) }\end{array}$ \\
\hline & & \multirow[t]{2}{*}{ iNO, SNAP } & & \multirow{2}{*}{$\begin{array}{l}\text { NO inhibits CVB3 replication in part } \\
\text { by inhibiting viral RNA and protein } \\
\text { synthesis }(7)\end{array}$} \\
\hline & & & & \\
\hline & & NO Donors & & $\begin{array}{l}\text { In vitro NO showed inhibition of the } \\
2 \mathrm{~A} \text { proteinase activity }\end{array}$ \\
\hline & & $\begin{array}{l}\text { SNAP, PFC, } \\
\text { GTN, } \\
\text { ISDN) }\end{array}$ & & $\begin{array}{l}\text { CVB3-infected mice showed } \\
\text { significantly reduced signs of } \\
\text { myocarditis after treatment with GTN } \\
\text { or ISDN (8) }\end{array}$ \\
\hline 22,23 & Influenza & $\begin{array}{l}\text { Gaseous } \\
\text { nitric oxide }\end{array}$ & In vitro & $\begin{array}{l}\text { Viral NA inhibition by gNO was } \\
\text { shown and may be responsible for }\end{array}$ \\
\hline
\end{tabular}




\begin{tabular}{|c|c|c|c|c|}
\hline & & \multicolumn{2}{|l|}{ (gNO) } & this antiviral effect (9) \\
\hline & & SNAP & & $\begin{array}{l}\text { inhibition of influenza virus viral RNA } \\
\text { synthesis (10) }\end{array}$ \\
\hline \multirow[t]{2}{*}{24} & $\begin{array}{l}\text { Japanese } \\
\text { encephalitis } \\
\text { virus (JEV) }\end{array}$ & SNAP & In vitro & $\begin{array}{l}\text { NO was found to profoundly inhibit } \\
\text { viral RNA synthesis, viral protein } \\
\text { accumulation, and virus release from } \\
\text { infected cells }(11)\end{array}$ \\
\hline & & $\begin{array}{l}\text { MDF to } \\
\text { produce } \\
\text { NO } \\
\text { (inducible } \\
\text { NO) }\end{array}$ & $\begin{array}{l}\text { In vitro \& } \\
\text { Murine } \\
\text { model }\end{array}$ & $\begin{array}{l}\text { MDF stimulated macrophages } \\
\text { inhibited virus replication with high } \\
\text { levels of NO production. MDF } \\
\text { treatment increased the survival rate } \\
\text { of JEV infected mice(12) }\end{array}$ \\
\hline 13 & Rhinovirus & $\begin{array}{l}\text { Nitric } \\
\text { Oxide } \\
\text { donor } \\
\text { (NONOate) }\end{array}$ & In vitro & $\begin{array}{l}\text { (NONOate) inhibited both rhinovirus } \\
\text { replication and cytokine production in } \\
\text { a dose-dependent fashion without } \\
\text { reducing levels of cytokine mRNA } \\
\text { (13) }\end{array}$ \\
\hline 6 & Reovirus & iNO & In vitro & $\begin{array}{l}\text { Cytostatic effects antiviral effects } \\
\text { e.g. reduction in DNA synthesis, } \\
\text { protein synthesis \& mitochondrial } \\
\text { metabolism(14) }\end{array}$ \\
\hline 9 & $\begin{array}{l}\text { Dengue virus } \\
\text { (DENV) }\end{array}$ & \multirow{2}{*}{ SNAP } & \multirow{2}{*}{ In vitro } & $\begin{array}{l}\text { NO showed an inhibitory effect on } \\
\text { viral RNA synthesis. The activity of } \\
\text { the viral replicase was suppressed } \\
\text { significantly (15) }\end{array}$ \\
\hline 10 & $\begin{array}{l}\text { Herpes simplex } \\
\text { virus type } 1 \text { (HSV } \\
\text { 1) }\end{array}$ & & & $\begin{array}{l}\text { Nitric oxide had inhibitory effects on } \\
\text { HSV1 protein and DNA synthesis as } \\
\text { well as on cell replication (16) }\end{array}$ \\
\hline 20 & $\begin{array}{l}\text { Porcine } \\
\text { circovirus type } 2 \\
\text { (PCV2) }\end{array}$ & $\begin{array}{l}\text { NO } \\
\text { generated } \\
\text { from } \\
\text { (GSNO) }\end{array}$ & $\begin{array}{l}\text { In vivo, In } \\
\text { vitro } \\
\text { (Murine } \\
\text { model) }\end{array}$ & $\begin{array}{l}\text { NO strongly inhibited PCV2 } \\
\text { replication in vitro. NO reduced the } \\
\text { progression of PCV2 infection in } \\
\text { mice(17) }\end{array}$ \\
\hline 11 & $\begin{array}{l}\text { Crimean Congo } \\
\text { hemorrhagic } \\
\text { fever virus } \\
\text { (CCHFV) }\end{array}$ & SNAP & In vitro & $\begin{array}{l}\text { NO reduced virion progeny yield } \\
\text { with reduction in expression of } \\
\text { viral proteins; the nucleocapsid } \\
\text { protein and the glycoprotein ,and } \\
\text { vRNA(18) }\end{array}$ \\
\hline 12 & $\begin{array}{l}\text { Respiratory } \\
\text { Syncytial Virus } \\
\text { (RSV) }\end{array}$ & $\begin{array}{l}\text { iNO, } \\
\text { SNAP }\end{array}$ & In vitro & $\begin{array}{l}\text { NO has significant direct antiviral } \\
\text { activity against RSV, which is more } \\
\text { potent with continuous, endogenous } \\
\text { NO production than exogenous NO } \\
\text { (19) }\end{array}$ \\
\hline 13 & $\begin{array}{l}\text { Human } \\
\text { papillomaviruses } \\
\text { (HPVs) }\end{array}$ & $\begin{array}{l}\text { NVN1000, } \\
\text { Topical } \\
\text { NO- } \\
\text { releasing } \\
\text { polymer }\end{array}$ & In vitro & $\begin{array}{l}\text { NO abrogated HPV- } 18 \text { progeny virus } \\
\text { production. Reduced HPV- } 18 \text { E6 and } \\
\text { E7 oncoproteins. Impaired S-phase } \\
\text { progression and induced DNA } \\
\text { damage in infected cultures (20) }\end{array}$ \\
\hline
\end{tabular}




\begin{tabular}{|c|c|c|c|c|}
\hline 14 & $\begin{array}{l}\text { Vesicular } \\
\text { stomatitis virus } \\
\text { (VSV) }\end{array}$ & iNO, SNAP & In vitro & $\begin{array}{l}\text { anti-VSV effects of NO in form of } \\
\text { significant inhibition of productive } \\
\text { VSV infection (21) }\end{array}$ \\
\hline \multirow[t]{3}{*}{15} & \multirow[t]{3}{*}{$\begin{array}{l}\text { Molluscum } \\
\text { contagiosum }\end{array}$} & \multirow{2}{*}{$\begin{array}{l}\text { Topical } \\
\text { acidified } \\
\text { nitrite, } \\
\text { nitric oxide } \\
\text { liberating } \\
\text { cream) }\end{array}$} & \multirow[t]{2}{*}{$\begin{array}{l}\text { A double- } \\
\text { blind, group- } \\
\text { sequential } \\
\text { clinical trial }\end{array}$} & $\begin{array}{l}75 \% \\
\text { cure rate in the active treatment } \\
\text { group }\end{array}$ \\
\hline & & & & $\begin{array}{l}\text { NO is effective therapy with } 75 \% \text { cure } \\
\text { rate in treatment group compared to } \\
21 \% \text { in control group (22) }\end{array}$ \\
\hline & & $\begin{array}{l}\text { topical } \\
\text { SB206 } \\
\text { (NO } \\
\text { releasing } \\
\text { topical } \\
\text { gel) }\end{array}$ & $\begin{array}{l}\text { multicenter, } \\
\text { randomized, } \\
\text { double- } \\
\text { blind, } \\
\text { vehicle- } \\
\text { controlled } \\
\text { clinical trial }\end{array}$ & $\begin{array}{l}\text { SB206 is effective therapy with } \\
\text { (SB206 } 12 \% \text { / once daily) provided } \\
\text { the best balance between MC lesion } \\
\text { clearance and tolerability (22) }\end{array}$ \\
\hline 17 & Hantavirus & iNO, SNAP & $\begin{array}{l}\text { In vitro, } \\
\text { murine } \\
\text { model }\end{array}$ & $\begin{array}{l}\text { NO strongly inhibited hantavirus } \\
\text { replication in vitro. The viral titers in } \\
\text { iNOS } \\
\text { to the contre were higher compared } \\
\text { inhibits hantavirus repling that NO } \\
\text { vivo(23) }\end{array}$ \\
\hline
\end{tabular}

Abbreviations: NO : Nirtic oxide, SNAP: S-nitroso-N-acetylpenicillamine, GTN: glyceryl trinitrate, ISDN: Isosorbide dinitrate, PFC: 4-phenyl-3-furoxancarbonitrile, iNO: inducible NO, CVB3: Coxsackievirus B3, gNO: Gaseous nitric oxide, NA: Neuraminidase, JEV: Japanese encephalitis virus, MDF: macrophage derived neutrophil chemotactic factor, NONOate: 3-(2-hydroxy-2-nitroso-1-propylhydrazino)-1propanamine, HSV1 :herpes simplex virus type 1, DENV: Dengue virus, PCV2: porcine circovirus type 2, GSNO: S-nitrosoglutathione, CCHFV: Crimean Congo hemorrhagic fever virus, RSV: Respiratory Syncytial Virus, VSV: Vesicular stomatitis virus.

\section{Figures}




\section{INHALED NO FOR CHEMOPROPHYLAXIS OF COVID19}

\section{SaNOtize (Inhaled NO)}

- Inhibits cellular protease termed Furin that accelerates canonical replication of SARS-COV 2 .

Decreases intracellular calcium that activates furin.

\section{USES:}

1- Early in the course of COVID-19

2- Post exposure chemoprophylaxis after documented exposure.

3- In healthcare personel at high

risk of exposure.

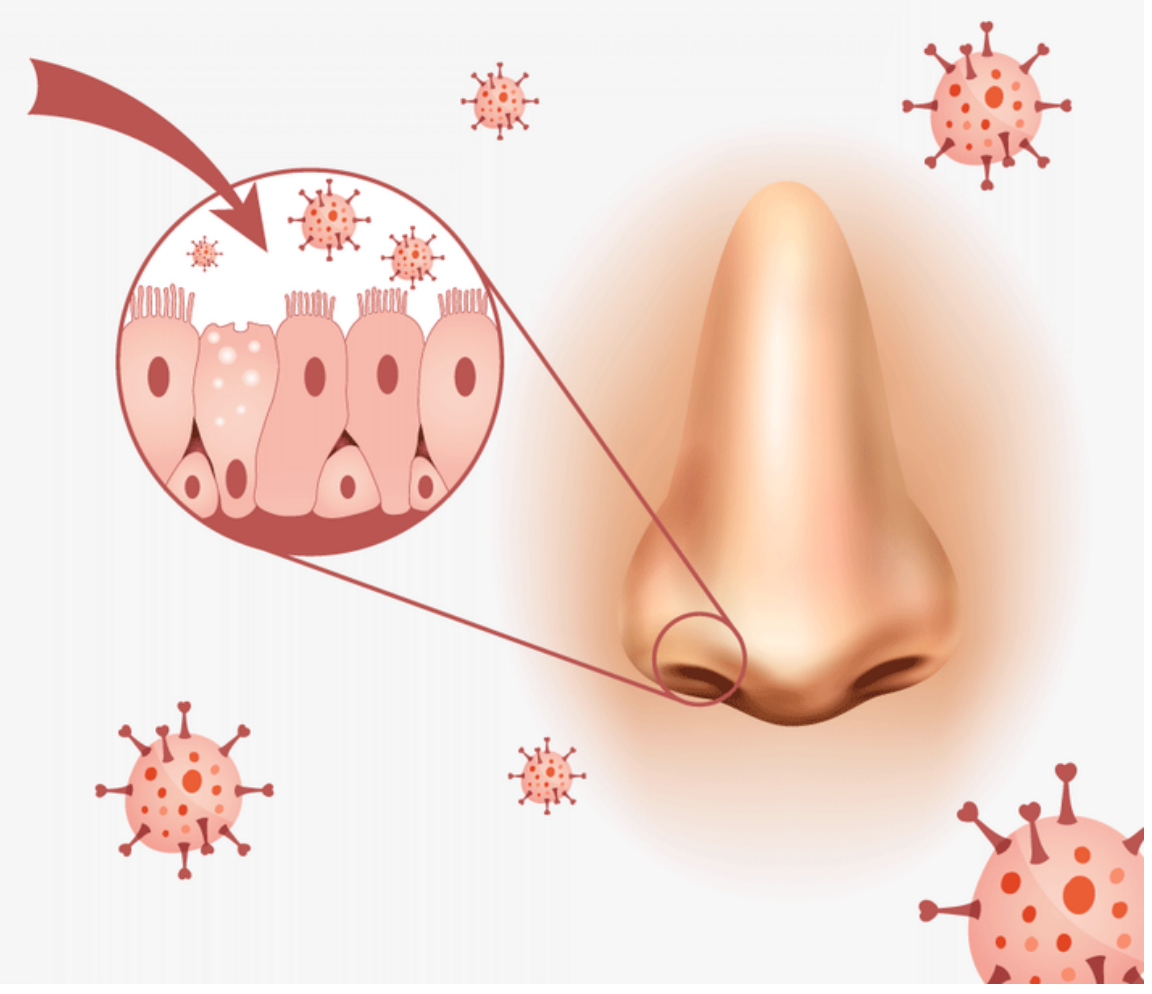

\section{Figure 1}

Title: Inhaled NO for chemoprophylaxis of COVID-19 Abbreviations: COVID-19: coronavirus 2019, NO, nitric oxide, SARS-CoV-2: Severe Acute respiratory syndrome coronaviridae-2 